\title{
Effectiveness of Tele-guided Interceptive Prosthodontic treatment in rural India: A comparative pilot study
}

\author{
Arun Keeppanasserril ${ }^{1}$, Anil Matthew ${ }^{1}$, Sapna Muddappa ${ }^{1}$ \\ ${ }^{1}$ Amrita School of Dentistry, Amrita Institute of Medical Sciences (AIMS), India
}

\section{Abstract}

Loss of teeth and resultant resorption of the residual ridges is a major oral health problem in India. The resorption leads to irreversible loss of bone volume of the jaws and seriously undermines retention and stability of future dentures. Loss of masticatory efficiency causes nutritional deficiencies and affects quality of life. However, construction of over-dentures (dentures anchored to modified teeth or roots), a sophisticated procedure requiring skills of several dental specialists, can arrest the resorption and provide retentive dentures. Dental specialists in India are, however, concentrated in urban areas leaving the rural populace under-serviced. The aim of our study was to find out whether newly graduated dentists, under remote guidance from specialists, can fabricate over-dentures that are functional and improve the oral health related quality of life.

Two groups of subjects were treated with over-dentures. Group 1 consisted of subjects attending a rural dental health clinic (site1) and group 2 at a university teaching hospital (site 2). Two dental graduates at each site carried out treatments. Operators at site 1 were guided remotely over a telemedicine link, cell phones, and emails while those at site 2 were guided directly. Functional assessment of dentures was carried out at the end of the treatment period to determine the technical quality of dentures. Subjective evaluation was carried out by subjects completing the Oral Health Impact Profile (OHIP-EDENT) questionnaire for edentulous subjects before and after treatment.

No statistically significant difference was seen between the functional assessment scores of dentures from the two sites $(p=0.08)$ at $95 \%$ confidence interval. Both groups also experienced significant improvement in all domains of OHIP - EDENT.

Remotely supervised newly graduated general dentists can provide over-dentures of sufficient quality to rural population. This strategy has the potential to improve access to care and elevate the level of dentistry available to rural population when referral to specialists in not feasible. The results of the study provide pointers for dental public health policy makers and administrators in developing nations on how to leverage Information and Communication Technology infrastructure to enhance access to care in rural areas.

Key words (MeSH):

General Practice, Dental/methods, Health Services Accessibility, Humans India, Remote consultation, Telemedicine, Denture, Overlay, General Practice, Dental/education*, Quality of life, Prosthodontics 
Effectiveness of Tele-guided Interceptive Prosthodontic treatment in rural India:

A comparative pilot study

\section{Introduction}

Edentulism is a major oral health problem in rural India. Although national statistics are not available, point prevalence studies have reported a prevalence rate of $72 \%(1)$. Loss of teeth triggers rapid onset of residual ridge resorption (RRR) which severely reduces retention and stability of any subsequent removable dentures(2). The functional and aesthetic sequelae of these events result in nutritional deficiencies and impaired quality of life $(Q O L)(3,4)(3,4)$. Construction of over-dentures anchored on few retained teeth can successfully reduce the rate of RRR and maintain functional efficiency of the masticatory system(5).

Construction of an over-denture is a sophisticated procedure requiring skills which are usually beyond general dentists (6). The expertise of a prosthodontist complimented by endodontists and oral surgeons is indispensable in fabricating them. Unfortunately most of the dental specialists in India are concentrated in urban areas leaving the semi urban and rural areas under served. The effect of geographical distance, socio-economic inequality and 'rural - urban' developmental imbalance on oral health care in India is profound. Less than twenty percent of the existing primary health centers in India have the services of a dentist available. At the moment India has one dentist for 10,000 persons in urban areas and one per 250,000 persons in rural areas. Almost three-fourths of the all the dentists are clustered in the urban areas, which is home to only onefourth of the country's population. It is often difficult for the urban poor and the rural population to get access to dental care (7).

Telemedicine has proved to be effective in overcoming geographical barriers and bridging the urban - rural healthcare divide in many parts of the world. Most of the instances of tele-medicine application in dentistry are restricted to diagnostic services and post-op follow-ups(8,9).In the only published instance of active dental treatment delivery via telemedicine linkage Berndt and Leone provided interceptive orthodontic treatment to underserved children in a rural community via telemedicine(10). There exists considerable scope for developing a similar strategy to extend prosthodontic treatment to rural areas, which do not have the services of dental specialists.

No comparable report of extending specialized prosthodontic services via telemedicine link is found in peer-reviewed journals. However, evidence suggests that general dentists, under close supervision, can satisfactorily perform a complex dental treatment like dental implants (6).

The present study aims to find out answer to the following question. Can minimally experienced general dentists guided remotely by prosthodontists make over-dentures that are functionally efficient and improve the oral health - related quality of life of patients when compared with over-dentures made by general dentists of similar experience?

\section{Methods}

A Cohort design was used for the study.

This study was conducted at two sites - a rural health centre (site 1) and the teaching Hospital of Amrita school of Dentistry in the city of Kochi in southern India. (site 2). Site 1 was connected to the University hospital periodically by satellite tele-medicine link and regularly by broadband Internet and cellphones.

Consecutive patients attending the dental clinics at both sites were screened for prosthetic need. 
Those who presented with less than 6 teeth in each arch were educated about over-dentures and approached for participation. A research assistant evaluated those who agreed to participate in the study to determine their eligibility as per the inclusion and exclusion criteria. Thirty subjects each were enrolled in the study at both sites. Both groups had similar selection criteria, treatment protocols, and outcome measures.

Two interns each were employed as the primary clinicians at both the sites. All of them had completed the 4-year Bachelor of Dental Surgery training and were undergoing the mandatory yearlong clinical rotation. They were given identical pre-training by the faculty at the university consisting of perusal of the office reference manual, shadowing the specialists, sitting in on lectures, pre-clinical simulation exercises and laboratory skill sessions. Operators at site 1 were guided remotely by scheduled video conferencing through satellite telemedicine linkage or broadband internet. In addition, cellphones were used sporadically to provide unscheduled guidance as and when required by the operators. Operators at site 2 were guided directly.

Apart from general medical and dental evaluation they were subjected to abutment evaluation and tentative jaw relation measurements. A blinded rater evaluated the case records to cases to ensure that the two groups were identical in complexity as much as possible.

Convenience sampling strategy was used for recruiting subjects in the study

The subjects were allowed to freely choose where they wanted to be treated according to their place of residence to enhance compliance.

Apart from general medical and dental evaluation the subjects were subjected to abutment evaluation and tentative jaw relation measurements.

Table 1: Inclusion and exclusion criteria

\begin{tabular}{|l|l|}
\hline Inclusion Criteria & Exclusion Criteria \\
\hline Stable general health & Unstable health. \\
\hline Healthy oral mucosa & Poor oral hygiene \\
\hline Normal jaw movements. & Teeth with gross coronal or root caries \\
\hline $\begin{array}{l}\text { No pronounced TMJ disorders } \\
\text { Availability of adequate denture space }\end{array}$ & $\begin{array}{l}\text { Unfavorable axial inclination of abutments } \\
\text { Poor crown root ratio. }\end{array}$ \\
\hline Availability of suitable abutments & Less than $7 \mathrm{~mm}$ alveolar bone support for abutments \\
\hline
\end{tabular}

The outcomes of interest in this study were

1)Functional quality of dentures

2)Self-reported Quality of life (QOL)

Denture quality was measured using the ten point Functional Assessment of Denture (FAD) criteria (11).

QOL was measured using a 19-point Oral Health Impact Profile (OHIP-EDENT) modified for 
denture - related quality of health. Although its construct validity and reliability have been validated the scale is not yet validated for Indian population. However, we chose the instrument because:

1. OHIP -14, a smaller subset of OHIP-EDENT has been validated in Indian population(12)

2. Absence of an oral health related QOL instrument for edentulous subjects validated in Indian population.

Functional evaluation of the dentures was conducted by two expert faculty not involved in the study. Subjects were required to complete OHIP-EDENT questionnaire translated in to the local language 'Malayalam' before the start of the treatment and at least 3 months after insertion of the dentures.

The institutional review board of Amrita School of Dentistry approved the study protocol. Express written consent was obtained from all the participants of the study.

Statistical analysis was performed by using SPSS V11.0 software. Paired sample testing was performed for OHIP-EDENT data. A t test was used to compare the FAD scores.

\section{Results}

Table 2: Demographic Information

\begin{tabular}{|l|l|l|}
\hline & Site I & Site II \\
\hline Patients with previous denture experience & 8 & 14 \\
\hline Minimum Age & 45 & 46 \\
\hline Maximum Age & 67 & 70 \\
\hline Average Age & 56 & 57 \\
\hline Number of Males & $86.7 \%$ & $73.3 \%$ \\
\hline Number of Females & 4 & 8 \\
\hline
\end{tabular}

Majority of the patients were males ( $86.7 \%$ and $73.3 \%$ at sites 1 and 2 respectively). Mean age of subjects at sites 1 and 2 was 56 and 57 respectively. The age range for site 1 was 45 to 67 while that of site 2 was 46 to 70 .

FAD scores of the two groups of the dentures fabricated at both the sites didn't exhibit statistically relevant difference. $(\mathrm{P}=0.08)$ (Table 3$)$ 
Effectiveness of Tele-guided Interceptive Prosthodontic treatment in rural India: A comparative pilot study

Table 3: Comparison of OHIP -EDENT scores before and after treatment - group (1)

\begin{tabular}{|l|l|l|l|l|}
\hline Domain & Subdomain & Mean & S.D & P-value \\
\hline \multirow{4}{*}{ Functional Limitation } & Chewing difficulty & 1.7000 & 1.51202 & $<0.001$ \\
\cline { 2 - 5 } & Food entrapment & 1.7000 & 1.23596 & $<0.001$ \\
\cline { 2 - 5 } & Ill-fitting denture & -0.7667 & 1.13512 & $<0.001$ \\
\hline \multirow{5}{*}{ Physical pain } & Painful aching in mouth & 0.2333 & 0.43018 & 0.006 \\
\cline { 2 - 5 } & Eating comfort & 1.7333 & 1.63861 & $<0.001$ \\
\cline { 2 - 5 } & Presence of sore spots & -2000 & 1.12648 & 0.339 \\
\cline { 2 - 5 } & Uncomfortable dentures & -0.4333 & 0.93526 & 0.17 \\
\hline \multirow{4}{*}{ Psychological discomfort } & Worry due dental problems & 0.7667 & 1.07265 & 0.001 \\
\cline { 2 - 5 } & self-conscious due to dental problems & 0.1667 & 0.69893 & 0.202 \\
\hline \multirow{3}{*}{ Psychological disability disability } & Avoiding some types of food & 1.8333 & 1.26173 & $<0.001$ \\
\cline { 2 - 5 } & Inability to eat & 0.3333 & 1.68836 & 0.288 \\
\cline { 2 - 5 } & Interruption to eating & 0.4667 & 1.56983 & 0.114 \\
\hline & Upset due to dental problems & 1.2333 & 1.88795 & 0.001 \\
\cline { 2 - 5 } & Embarrassed due to dental problems & 1.0667 & 1.70057 & 0.002 \\
\hline \multirow{3}{*}{ Social disability } & Avoid going out & 0.4000 & 1.30252 & 0.103 \\
\cline { 2 - 5 } & Less tolerant with friends and family & 0.3333 & 0.92227 & 0.057 \\
\cline { 2 - 5 } & Irritable to others & 0.1000 & 0.95953 & 0.573 \\
\hline \multirow{3}{*}{ Handicap } & Unable to enjoy company & 0.3333 & 0.66089 & 0.01 \\
\cline { 2 - 5 } & Dissatisfaction with life in general & 0.7000 & 1.57896 & 0.022 \\
\hline
\end{tabular}

Comparison between the OHIP -EDENT scores before and 3 months after treatment revealed that subjects in both groups exhibited significant improvement in all the domains. Overall, 11 out of 19 subdomains showed improvement. (table 3 and table 4). 
Effectiveness of Tele-guided Interceptive Prosthodontic treatment in rural India: A comparative pilot study

Table 4: Comparison of OHIP -EDENT scores before and after treatment - group 2

\begin{tabular}{|l|l|l|l|l|}
\hline \multirow{5}{*}{ Functional Limitation } & Subdomain & Mean & S.D & Pvalue \\
\cline { 2 - 5 } & Chewing difficulty & 1.7333 & 0.2793 & $<0.001$ \\
\cline { 2 - 5 } & Food entrapment & 1.8333 & 0.20945 & $<0.001$ \\
\cline { 2 - 5 } & Ill-fitting denture & -0.2667 & 1.17248 & 0.223 \\
\hline Physical pain & Painful aching in mouth & 1.167 & 0.20945 & $<0.001$ \\
\cline { 2 - 5 } & Eating comfort & 1.700 & 0.33614 & $<0.001$ \\
\cline { 2 - 5 } & Presence of sore spots & -0.100 & 0.23659 & 0.676 \\
\cline { 2 - 5 } & Uncomfortable dentures & -0.4 & 0.81368 & 0.012 \\
\hline Psychological discomfort & Worry due dental problem & 1.3667 & 1.24522 & $<0.001$ \\
\cline { 2 - 5 } & Self-conscious due to dental problem & 0.4333 & 0.77385 & 0.005 \\
\hline Physical disability & Avoiding some types of food & 1.8667 & 1.45586 & $<0.001$ \\
\cline { 2 - 5 } & Inability to eat & 0.5333 & 1.71672 & 0.100 \\
\cline { 2 - 5 } & Interruption to eating & 0.931 & 1.70987 & 0.007 \\
\hline Psychological disability & Upset due to dental problem & 1.4667 & 1.85199 & $<0.001$ \\
\cline { 2 - 5 } & Embarrassed due to dental problem & 1.1333 & 1.52527 & $<0.001$ \\
\hline Social disability & Avoid going out & 0.6333 & 1.15917 & 0.006 \\
\cline { 2 - 5 } & Less tolerant with friends and family & 0.6 & 1.30252 & 0.017 \\
\cline { 2 - 5 } & Irritable to others & 0 & 0.98261 & 1.000 \\
\hline \multirow{5}{*}{ Handicap } & Unable to enjoy company & 0.1333 & 0.9732 & 0.459 \\
\cline { 2 - 5 } & Dissatisfaction with life in general & 0.6 & 1.16264 & 0.008 \\
\hline
\end{tabular}

\section{Discussion}

We evaluated the two major factors that reflect on success of prosthodontic treatment: 1 . Functional efficiency of the denture as obtained by clinical evaluation 2. Oral health related quality of life (OHRQOL) as reported by the subjects. Successful oral rehabilitation with dentures depend on both technical factors and patients' subjective perceptions (12). A sizeable volume of research identifies OHRQOL as a predictor of successful dentures (13-15). No statistically significant difference was observed between the FAD scores of the two groups of patients. Both groups also showed significant improvements in almost all domains of OHRQOL as measured by OHIP -EDENT. These results are consistent with those from Estafandiari et al and $\mathrm{J}$ Brendt et al $(6,9)$.

Teledentistry, the extension of telemedicine to include dental services has the potential to "fundamentally change the way dental care is delivered today" (16). The results of the present study have important implications for specialized dental treatment delivery in rural areas of developing nations. Employing new graduates receiving real-time guidance from experts located at an urban center may mitigate the shortage of specialist dental practitioners in such areas. This approach has the potential to improve access to care and the level of dental care available to rural population.

Training of the general dentists, hardware and software problems, poor network, and patient 
compliance were few of the problems encountered during our study. A high degree of unreliability in connectivity and the need for on-site information-technology support often presented hurdles and led to several appointments at site 1 being extended or even rescheduled. We postulate that rapid improvements in telecommunication infrastructure coupled with improved hardware availability and reduction in cost will significantly reduce these challenges in future. Transportation issues leading to missed appointments, poor oral hygiene maintenance, and the not-so-rare lack of family support for the long treatment sequence also presented problems.

\section{Limitations}

The study has several limitations which need to be considered in interpreting the results. a. Limitations in the choice of study design - Randomized controlled trials (RCT) are considered the Gold Standard for establishing the effectiveness of clinical interventions and hence would have been the ideal study design. Randomization was difficult to achieve because of the geographic distance between the two sites. Construction of overdentures requires multiple appointments over a series of visits. Both these factors made true randomization impractical. Patient compliance issues also were considered during the design phase of the study. Cross over design was considered but the irreversible nature of tooth preparation ruled out its choice.

b. Limitation in the sample - Number of subjects in the study had to be limited due to financial, technical and logical constraints. No attempt was made to match the two groups of patients with each other, which reduces external validity of the results.

Absence of an economic evaluation is another drawback of the study from policy and sustainability point of view.

\section{Policy Recommendations}

Innovative use of technology and manpower can aid communities in overcoming traditional shortcomings where there is disparity of healthcare access. The present study used a model in which available communication infrastructure was used to extend quality dental treatment to underserviced areas. The model has the potential for significant impact in developing nations like India with vast tracts of rural and semi urban areas with little or no access to specialty dental care. In several regions of the developing world recent improvements in communication infrastructure has opened up a promising avenue to improve healthcare in previously inaccessible areas. The cost of communication services and required equipment are on a downward trend while the available bandwidth is progressively increasing. It is recommended that until such time as physical extension of facilities and manpower are made public health authorities may consider extending the existing manpower and expertise by using telehealth networks. In situations where the use of telemedicine equipment is not feasible it can be substituted by using available broadband Internet connections. At the same time, care must be taken to ensure that these services are integrated with the existing health care system. The ideal strategy would be consider factors like cost, accessibility, social isolation, poverty, and to customize the solution to suit local needs. It is a common trend to consider telehealth just as an add-on service. Such an approach might result in under realization of its full potential. It is advised to consider telehealth services such as those described in the present articles as potentially high impact interventions, and accorded sufficient attention. Legal, ethical, and confidentiality issues also need to be considered 
Effectiveness of Tele-guided Interceptive Prosthodontic treatment in rural India: A comparative pilot study

while formulating policies. Adequate levels of documentation of care as well as technical logs are vital for continued improvement of the services and hence made mandatory.

\section{Conclusion}

Despite some limitations, this study provides definite indications that pre-trained new dental graduates can, when supervised remotely by specialists, provide tooth supported over-dentures of satisfactory quality. This suggests that telemedicine has the potential to improve access to quality dental care for rural populace.

\section{Acknowledgments}

Amrita Institute of Medical Sciences permitted use of telemedicine infrastructure and support personnel. Dental materials and equipment were provided by Amrita School of Dentistry. No conflict of interest.

\section{References}

(1)Prabhu N, Kumar S, D'Souza M. Partial edentulousness in a rural population based on Kennedy's classification: an epidemiological study. Journal of Prosthodontic Society; 2009 ; 9(6) : 18-23

(2)Atwood D. Reduction of residual ridges: a major oral disease entity. The Journal of Prosthetic Dentistry. 1971 Sep;26(3):266-79

(3)Akifusa S, Soh I, Ansai T, Hamasaki T. Relationship of number of remaining teeth to health-related quality of life in community-dwelling elderly. Gerodontology: 2005; Jun; 22(2): $91-7$

(4) McGrath C. Can dentures improve the quality of life of those who have experienced considerable tooth loss ? Journal of Dentistry. 2001; May; 29(4):243-6

(5)Fenton A.The decade of overdentures: 1970-1980. The Journal of Prosthetic Dentistry.1998; Jan; 79(1): 31-36

(6)Esfandiari S, Lund J, Thomason J, Dufresne E, Kobayashi T, Dubois M, et al. Can general dentists produce successful implant overdentures with minimal training ? Journal of Dentistry. 2006 Nov.; 34(10):796-801

(7)Shobha Tandon. Challenges to the Oral Health Workforce in India J Dent Educ 2004 68(7): 28-33

(8)Berndt J, Leone P. Using teledentistry to provide interceptive orthodontic services to disadvantaged children. American Journal of Orthodontics and Dentofacial Orthopedics 2008; 2008 Nov; 134(5):700-706

(9)Anastassiadou V, Naka O, Heath M. Validation of indices for functional assessment of dentures. Gerodontology. 2002; Jul; 19(1): 46-52

(10)Acharya S. Oral health-related quality of life and its associated factors in an Indian adult population. Oral Health Preventive Dentistry. 2008; 6(3):175-184

(11)De Lucena SC, Gomes SGF, Da Silva WJ, Del Bel Cury AA. Patients' satisfaction and functional assessment of existing complete dentures: correlation with objective masticatory function. Journal of Oral Rehabilitation. 2010 Oct. 29; 38(6): 440-446 
(12)Heydecke G, Locker D, Awad M. Oral and general health-related quality of life with conventional and implant dentures. Community Dentistry and Oral Epidemiology; 2003 Jun; 31(3):161-168

(13)Allen P. The impact of tooth loss in a denture wearing population: an assessment using the Oral Health Impact Profile. Community Dental Health.1999 Sep; 16(3): 176-80

(14)Bouma J, Boerrigter L, Van Oort R. Psychosocial effects of implant-retained overdentures. International Journal of Oral \& Maxillofacial Implants.1997; Jul-Aug; 12(4): 515-22

(15)Cooper B. Knowledge, attitudes, and confidence levels of dental hygiene students regarding teledentistry: A pilot study. The Internet Journal of Allied Health. 2007 Oct; 5(4) 1- 4

(16)Lewis D. Optimized therapy for the edentulous predicament: cost-effectiveness considerations. The Journal of Prosthetic Dentistry. 1998 Jan;79(1):93-99 